\title{
The impact of measures when optimising sales processes using Scrum
}

\author{
Article by Riaan Steenberg \\ PhD, Texila American University, South Africa \\ Email: riaan.steenberg@gmail.com
}

\begin{abstract}
The author used the Scrum management tool to optimise the processes of two sales teams. Different types of measures were used in two business units to define success in an agile improvement setup over 21 weeks. The team that focused on calls and activities performed better both quantitatively and qualitatively than the team that tracked leads and focused on financial values of contracts. The paper concludes that activity based measures is a precondition for success in a sales environment that will utilise SCRUM as an optimisation method.
\end{abstract}

Keywords Sales, Scrum, Measurement, Performance, Improvement

\section{Introduction}

One of the promises of SCRUM as a management method is that processes can be optimised through a persistent focus on the process constraints and clear process measures as the output of a system.

Scrum emerged as a management methodology, typically applied in a software environment, by creating a burn down on a set of features towards a release date.

Van Soligen, Sutherland and De Waard (2011) suggests that the use of SCRUM as a management method in sales, and in various other disciplines can benefit form the benefits given by SCRUM which include:

- Value prioritization;

- Self-steering, teams;

- Joint task definition; and

- Continuous improvement.

The author used the Scrum process as a tool to optimise the processes of two sales teams.

Different measures were used in a business to consumer sales team and a business-tobusiness sales team due to their apparently different nature. The team that focused on calls and quantities performed better than the team that tracked leads and focused on financial values of contracts.

The intent of this study is to establish a well-defined framework to inform the choice of measures that will be relevant in the use of SCRUM as a management method in a sales environment.

\section{Methodology}

In the specific company we investigated, there are a number of sales teams. Two teams were of particular interest as they focused on the same essential sales process, being outbound sales while one had a specific focus on retail or B2C and the other had a focus on corporate or B2B sales.

Over time each team had evolved a different mind-set in relation to the goals they set and reported differently on their sales progress.

The retail team specifically reported on activity based measures such as:

- Number of calls; and

- Number converted.

The corporate sales team more specifically reported on

- Deal sizes; 
South American Journal of Management

Special Edition 2016

- Stage of conversion; and

- Values of deals.

A sales target was set for each team. Daily progress on the measures that were established was measured with the facilitator focusing on ways to improve the constraints that were creating real or perceived constraints in the sales team.

The purpose of the study was to investigate the significance of specific measures in determining the performance of a sales team, both of which uses a Scrum methodology for constant improvement.

This is motived by the conference paper presented by Van Solingen, Sutherland and De Waard (2011) that stated the application of SCRUM to the sales environment, seems to be an important area of study and application. They noted that their study was the first formal documentation of the use of Scrum in the sales environment.

Teams were actively engaged in daily SCRUM meetings facilitated by an experienced sales manager that completed three core process activities.

1) Review of metrics

2) Asking scrum questions that included

a. What did we achieve yesterday

b. What do we need to do to change the game

c. What do we need to achieve today

3) There was then specific focus given to the actions that were identified as constraints or opportunities during the day including giving feedback to marketing, finance and other teams that may be impacted by specific sales initiatives.

Results on metrics were monitored through self-disclosure with occasional check back on actual statistics from other systems to verify the accuracy of claims. This is significant, as each person "owned" his or her own metrics.

Seven (7) cycles of three (3) weeks each were completed and the measurements below are presented over a 21 -week period.

\section{Results and Findings}

The table below presents the change in key metrics between the two teams over a 21 -week period.

\begin{tabular}{|c|c|c|c|}
\hline \multicolumn{4}{|l|}{21 Week Metrics Summary } \\
\hline Metric & & & \\
\hline Retail Team & Starting & Ending & Change \\
\hline Outbound Calls & 2 & 25 & $1150 \%$ \\
\hline Inbound Calls & 25 & 40 & $60 \%$ \\
\hline Conversion conversations & 2 & 15 & $650 \%$ \\
\hline Deals converted per week & 17 & 27 & $59 \%$ \\
\hline Conversion Ratio (Per Week) $\left(^{*}\right)$ & $13 \%$ & $8 \%$ & $-34 \%$ \\
\hline Weekly Revenues $(*)$ & $1,700,000$ & $2,700,000$ & $59 \%$ \\
\hline Annualised revenue & $68,000,000$ & $108,000,000$ & $59 \%$ \\
\hline \multicolumn{4}{|l|}{ Corporate Team } \\
\hline Number of clients & 205 & 76 & $-63 \%$ \\
\hline Pipeline revenue & $24,000,000$ & $125,000,000$ & $421 \%$ \\
\hline Total Conversion & $5,000,000$ & $15,000,000$ & $200 \%$ \\
\hline
\end{tabular}




\begin{tabular}{|l|l|l|l|} 
Weekly conversion $(*)$ & $1,325,000$ & 714,286 & $-46 \%$ \\
\hline Conversion Ratio $(*)$ & $5.521 \%$ & $0.571 \%$ & $-90 \%$ \\
\hline Annualised Revenue $(*)$ & $53,000,000$ & $28,571,429$ & $-46 \%$ \\
\hline
\end{tabular}

(*) Not measured by SCRUM teams

Figure 1. 21 Weeks Metrics Summary as at start and end for two teams

It must also be noted that there was no change in team size or any other variables during the duration of the study.

The retail team was much more effective as there was better activity and it is postulated that this led to better results. For the retail team, management was focused on improving activity and conversion ratios the optimization that was done improved these metrics. Management asked the questions on why activity was not optimized and this led to a slew of improvements in processes, layouts, materials and constraints that impacted on the activity levels of sales people.

From the data it can be seen that most metrics were affected positively, however it can be noted the conversion ratio in both teams decreased. This mediates the result as higher levels of activity may not result in the process leading to more conversions but may result in a faster conclusion on the potential of a client and a higher sales velocity. It must be noted that during the 21 week cycle that the conversion ratio was not discussed or optimized as part of the scrum process.

For the corporate team, management was focused on growing the size and quantity of potential deals and tracking the stage of progress in the "pipeline". Many companies that were on the pipeline turned out not to want to buy and this created a shakeout. In the end conversion ratios became a lot smaller but in a sense more predictable. Management was focused on finding more companies to target and on improving methods to target them, while sales people were more concerned with estimating the size and value of the potential deal and less on the activity levels per stage.

The comparison of the results then asks the question: if the measures that were chosen would be more effective in determining the relative outcome of sales and that the inherent optimization that is postulated by a methodology based on the SCRUM management method, would work better on activity based measures in a sales environment than process based measures.

\section{Discussion/Conclusion}

\section{What must sales measure?}

The issue of what to measure in sales is still an area without definitive results. Moutot and Bascoul (2008) created a framework that showed there are direct linkages between sales calls and proposal generation on the key success determinants for sales that was relationship initiation and relationship maintenance. They also showed that focusing on quality (successful sales calls) and converted proposals lead to earlier relationship termination, which ultimately is expected to lower costs of attempting to convert non-profitable customers. This focus on quality practices makes sure that sales activity is more efficient.

By focusing on improving sales force planning and configuration, the Scrum methodology would thus have a higher relationship initiation and relationship maintenance focus. The choice of metrics in the Scrum process can lead to increase in relationship terminations as the choice of metrics influences the determination of "success" within the sales environment. This was the case in the corporate sales team as more clients were eliminated earlier during the process.

Jordan and Vazanna (2011) outlines that sales forces have four type of metrics 
South American Journal of Management

Special Edition 2016

\begin{tabular}{|c|c|}
\hline $\begin{array}{c}\text { Sales Force } \\
\text { Capability }\end{array}$ & $\begin{array}{l}\text { Market Coverage } \\
\text { Developing a sales force that is able to } \\
\text { cffectivcly exceute its sclling capabilitics }\end{array}$ \\
\hline Customer focus all desired opportunitics
\end{tabular}

Figure 2. Scope of sales metrics

The Scrum methodology would naturally focus on enhancing sales force capability and highlight issues with customer and product focus.

Another perspective on sales metrics is that if a clear win/loss (activity) ratio is established, any sales process can be made "predictable". By focusing on the standardized process that leads to a specific win/loss ratio it can create clear stages through which a customer must go.

Through having this clear view of the sales process, this allows for the remainder of the sales activities to be optimized. Scrum can mediate this scenario through asking questions that outline what the factors are that contribute to higher win/loss ratios and assisting in sales force planning towards optimizing this ratio.

This is depicted in Table 3 which highlights that a potential prospect goes through a process based view of sales, which results in a converted sale. The SCRUM process would thus contribute to the process optimization by focusing on improving the activities in each of the process steps and thus leading to both a more productive and capable sales team.

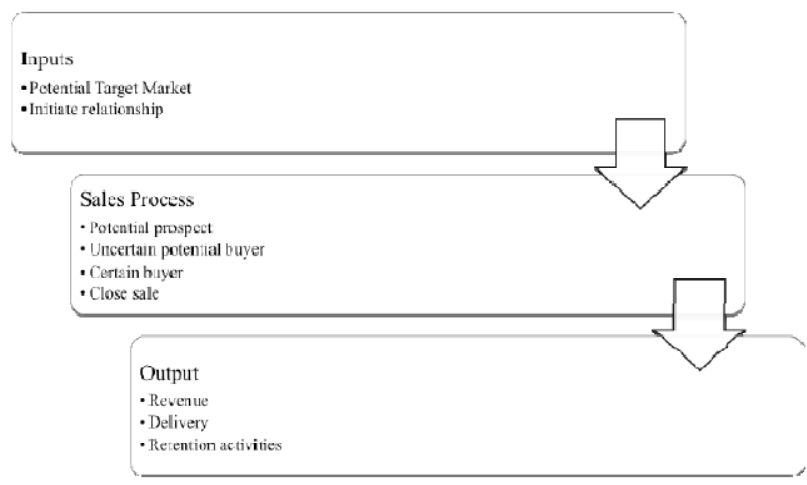

Figure 3. A sales funnel approach to sales conversion

\section{Why Scrum?}

SCRUM as a management method is that processes can be optimised through a persistent focus on the process constraints and clear process measures as the output of a system.

Sales is a system that takes a long time to perfect and is highly dependent on creating feedback on what customers want in the business.

Scrum was originally designed as a feedback system that creates multiple interaction points to creating shippable software within faster release cycles. The benefit of this approach in software development is that each phase or cycle has a clear and tangible output and that there is a focus on the most important aspect of the product development at any point in time.

They key characteristics of a scrum

- Self-organizing teams;

- Product progresses in a series of time-defined "sprints"; 
- Requirements are captured as items in a list of "product backlog";

- A central goal defines a "sprint";

- Short (15 minute) daily stand-up and check-in meetings are used to give feedback on progress and monitor results;

- Each sprint is reviewed in a "retrospective" meeting that captures learning and sets goals for the next sprint.

- Every day a specific set of tasks are selected for focus towards the goal;

- Constraints are identified and actions implemented to remove these constraints;

- Uses generative rules to create an agile environment for delivering projects; and

- One of the "agile" processes.

Scrum works in sales where there is a need to define clearly what customers want and how to execute this better. It gives constant feedback to sales people on how they are doing in relation to goals and sets up competition and collaboration towards increasing effectiveness. "Plateaus" in sales can be addressed by creating minor incentives for breaking through to a next level and learning from one person or group can be applied to a larger group.

This creates a dynamic and vibrant environment where employees feel that there are administrative and procedural justice and that their input in valued. These factors combine to a healthy socio-technical environment that is one of the pre-conditions to a high-performance team.

\section{Scrum and sales measures}

This study highlights that the choice of measures in a scrum system is critical and that choosing activity based measures in a sales environment is likely to ensure that the factors that is in the control of the sales person is addressed. Focusing purely on pipeline and growth of sales potential is not adequate and creates a set of measures that reward the incorrect behaviour of sales people.

It is likely that a focus on conversion ratio is very critical measurement in this environment and that an increased focus on higher conversion would highlight why potential customers do not want to buy and what can be done to convert these more effectively.

\section{Limitations}

This is a small study of two teams over a 21 -week period. What makes it unique is that various variables could be eliminated over the period due to the similarity of focus and development of both teams in a similar environment.

\section{Future directions}

A more comprehensive study with multiple measures and their relevance to the scrum method may yield a more comprehensive view of relevant measures and how they interact. Already the study has identified that a likely focus on activity measures with conversion ratios would yield positive impacts and identify more pertinent issues.

It would also be meaningful to test this in other sales environments to see if results are consistent.

\section{References}

[1.] Jordan, J., \& Vazzana, M. (2011). Cracking the Sales Management Code: The Secrets to Measuring and Managing Sales Performance.

[2.] Moutot, J. M., \& Bascoul, G. (2008). Effects of sales force automation use on sales force activities and customer relationship management processes. Journal of Personal Selling \& Sales Management, 28(2), 167-184.

[3.] Van Solingen, R., Sutherland, J., \& De Waard, D. (2011, August). Scrum in sales: How to improve account management and sales processes. In Agile Conference (AGILE), 2011 (pp. 284-288). IEEE. 